\title{
Microfluidic Chain Reaction: Conditional, Structurally Programmed Propagation of Capillary Flow Events
}

Mohamed Yafia, ${ }^{1,2, \dagger}$ Oriol Ymbern, ${ }^{1,2, \dagger}$ Ayokunle O. Olanrewaju ${ }^{1,2, \S}$, Azim Parandakh ${ }^{1,2}$, Ahmad $^{2}$ Sohrabi Kashani ${ }^{1,2}$, Johan Renault ${ }^{1,2}$, Zijie Jin ${ }^{1,2}$, Geunyong Kim ${ }^{1,2}$, Andy $\mathrm{Ng}^{1,2}$, David Juncker ${ }^{1,2, *}$

1. Biomedical Engineering Department, McGill University, Montreal, QC, Canada

2. McGill Genome Centre, McGill University, Montreal, QC, Canada

${ }^{\dagger}$ Equal contribution. ${ }^{\S}$ Current address: Mechanical Engineering Department, University of Washington, Seattle, WA, USA.

\section{Abstract:}

Chain reactions are characterized by initiation, propagation and termination, are stochastic at microscopic scales and underlie vital chemical (e.g. combustion engines), nuclear and biotechnological (e.g. polymerase chain reaction) applications. ${ }^{1-5}$ At macroscopic scales, chain reactions are deterministic and limited to applications for entertainment and art such as falling domino and Rube Goldberg machines. Appositely, the microfluidic lab-on-a-chips (also called a micro total analysis system), ${ }^{6,7}$ was envisioned as an integrated chip, akin to microelectronic integrated circuits, yet in practice remain dependent on cumbersome peripherals, connections, and a computer for automation. ${ }^{8-11}$ Capillary microfluidics integrate energy supply and flow control onto a single chip by using capillary phenomena, but programmability remains rudimentary with at most a handful (eight) operations possible. ${ }^{12-19}$ Here we introduce the microfluidic chain reaction (MCR) as the conditional, structurally programmed propagation of capillary flow events. Monolithic chips integrating a MCR are 3D printed, and powered by the free-energy of a paper pump, autonomously execute liquid handling algorithms step-by-step. With MCR, we automated (i) the sequential release of 300 aliquots across chained, interconnected chips, (ii) a protocol for SARS-CoV-2 antibodies detection in saliva, and (iii) a thrombin generation assay by continuous subsampling and analysis of coagulation-activated plasma with parallel operations including timers, iterative cycles of synchronous flow and stopflow operations. MCRs are untethered from and unencumbered by peripherals, encode programs structurally in situ, and can form frugal, versatile, bona fide lab-on-a-chip with wide-ranging applications in liquid handling and point-of-care diagnostics. 


\section{Main:}

The MCR encodes the deterministic release of reagents stored in a series of reservoirs, with the release of reservoir $n$ being conditional on emptying (draining) of the reagent in reservoir $n-1$, and emptying reservoir $n$ in turn triggering the release of reservoir $n+1$. Capillary domino valves (CDVs) encode this condition, and serially connect, i.e. chain, the reservoirs, and thus control the propagation of the chain reaction (Fig. 1a). MCRs were implemented in 3Dprinted circuits made with a common stereolithography printer with feature size from $100 \mu \mathrm{m}$ to $1.5 \mathrm{~mm}$, hydrophilized using a plasma (see Supplementary Fig. S1 and S2), sealed with a plain cover, and connected to a capillary pump made of paper (filter papers or absorbent pads). The paper is spontaneously wetted by aqueous solution drawn from the microfluidic circuit by releasing free energy stored in the paper surface, and drives the chain reaction; expressed differently, the capillary pump generates a negative capillary pressure that is hydraulically transmitted back into the circuit via the main channel and serially drains side-reservoirs connected via a small conduit, called functional connection (further described below). CDVs form air links between adjacent reservoirs, serially connecting them along a path parallel to the main channel, but interrupted by filled reservoirs that form liquid plugs between CDV air links. When the (first) reservoir connected to the air vent via a continuous air link is emptied, the plug is removed, and the length of the air link propagates to the next filled reservoir in the MCR (Fig. 1a-d, and Video S1). This simple design structurally encodes the conditional propagation of capillary flow events and the step-by-step release of an arbitrary number $N$ of reservoirs without peripheral connection or moving parts, and is further detailed in the supplementary information.

MCR require ancillary capillary microfluidic components that fulfill different functions depending on the intended operation (e.g. loading, holding, mixing, and draining liquids following the MCR progression) to form fully integrated and scalable capillaric circuit (CCs). CCs are designed based on a library of building blocks including capillary pumps, flow resistances, and many types of capillary valves (stop v., trigger v., retention v., retention burst v.), ${ }^{12,14}$ and thus analogous to microelectronic integrated circuits, but lacking scalability and functionality theretofore. In MCRs, samples are loaded by capillary flow via an inlet with a capillary retention valve and entirely fill the reservoirs lined with three stop valves, including two with a dual retention burst valve function connecting to the two lateral CDVs, and one at the intersection of the functional connection and the main channel (Fig. 1c). Although the functional 
1 connection is a deceptively simple straight channel, it fulfills six key functions. It is (i) the air

2 vent during filling of the reservoir, and (ii) a stop valve preventing the reagent from spilling into

3 the main channel while it is empty. After filling of the main channel, it forms a (iii) hydraulic

4 link propagating the pressure from the main channel into the reservoir and (iv) a barrier (and

5 bottleneck) to the diffusion of reagents between the reservoir and the main channel. (v) It becomes the outlet and a flow resistance (discussed further below) during reservoir emptying, and (vi) a capillary retention valve stopping air from invading the main conduit after the reservoir is emptied. As a result, many trade-offs guide its design.
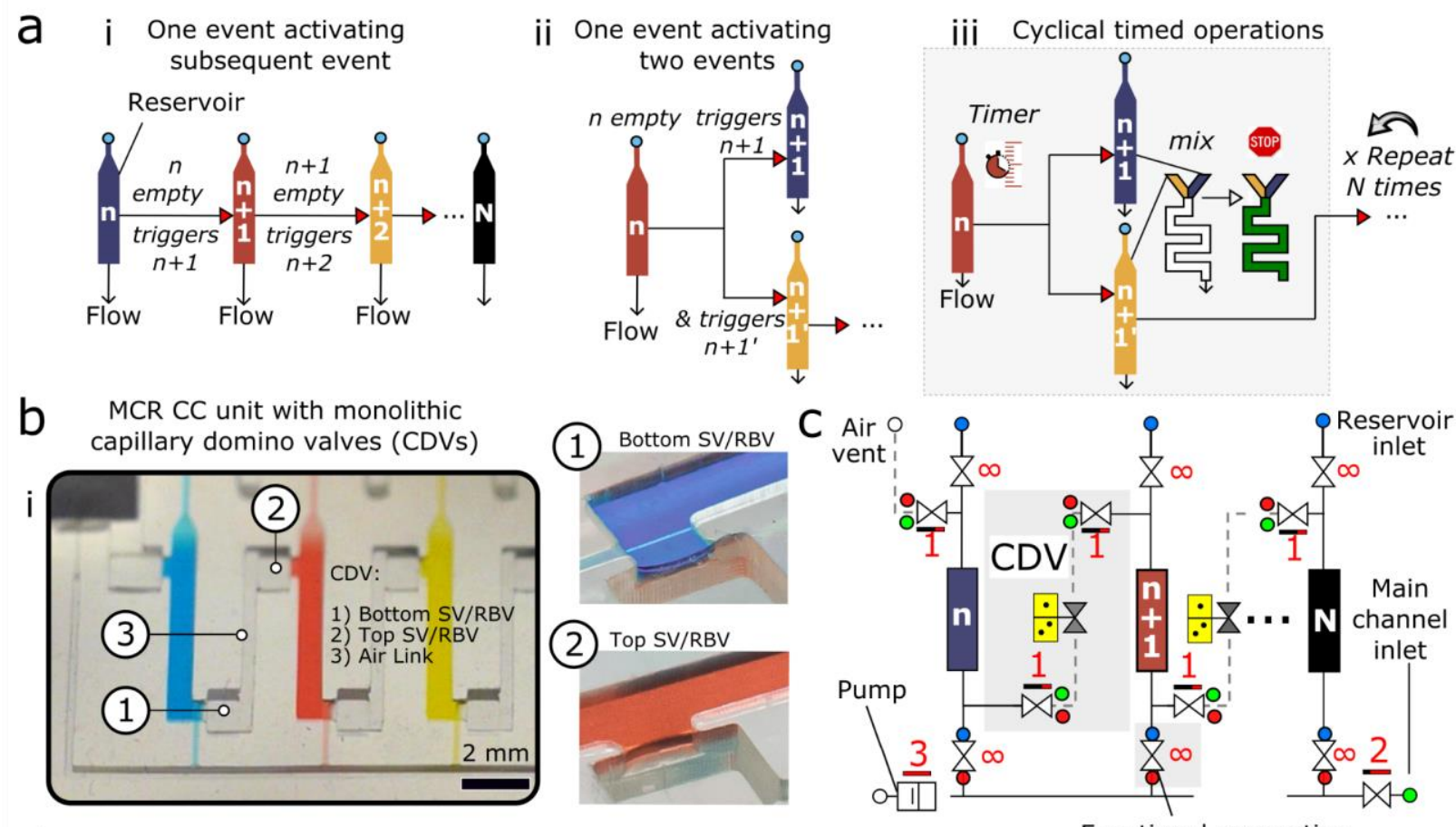

- Stop valve (SV) ○ Retention burst valve (RBV) • Capillary retention valve (CRV)
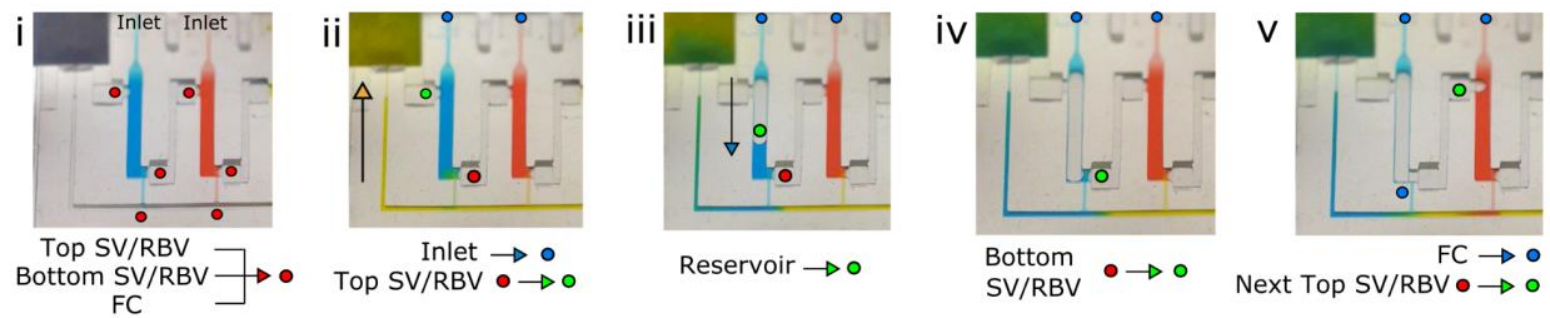

Fig. 1. Microfluidic chain reaction (MCR) with monolithic capillary domino valve (CDV) for serial delivery of reagents in capillaric circuits (CCs). (a) i- Serial MCR, ii- Branching MCR, iii- Cascaded, timed MCR. (b) MCR unit with 3 reservoirs chained via CDVs and closeup of dual function SV/RBVs (stop valve/retention burst valve) that keep liquid out of the CDV 
air link (forming a pneumatic connection) and prevent premature drainage. (c) Symbolic view of the MCR unit with capillary retention valves (' $\infty$ ' symbol), CDV (grey overlay) that includes an air link, two SV/RBVs and functional connection (FC). (d) Screen shots of Video S1 showing MCR sequences where most of the capillary elements have dual functions, one during reagent loading, one during MCR propagation. i- A loaded chip with liquids confined to the reservoirs by physical and capillary valves. ii- MCR is triggered (the inlet becomes a capillary retention valve and the top SV becomes a RBV). iii- Emptying of the first reservoir upon bursting of the top RBV. iv- The bottom SV momentarily becomes an RBV that bursts immediately. v- Air now occupies the emptied reservoir. The FC becomes a capillary retention valve preventing the air from penetrating into in the main channel. The air link connects the air to the RBV of the next reservoir, which bursts, and triggers reservoir emptying.

We sought to understand the design window and failure modes of MCR, notably under which conditions downstream of CDVs might trigger prematurely, using both theory and experiments. MCR-CCs incorporate numerous capillary stop valves according to previously established design criteria ${ }^{13}$ and while considering $3 \mathrm{D}$ printer performance including resolution, imprecision, and printing errors. We then analyzed the MCR based on an electrical circuit analogy, Supplementary Fig. S3, and derived a simplified circuit that neglects minor resistances, Fig. 2a. ${ }^{13}$ Successful and incremental propagation of the MCR is conditional on preventing the breach of the liquid in reservoir $n$ into the CDV and air link connecting $n+1$, which is equivalent to stating that all the liquid in reservoir $n$ must flow exclusively through the functional connection $n$.

The flow path from reservoir $n$ to $n+l$ is interrupted by the CDV, which includes the capillary stop valve at one extremity and retention burst valve at the other, with bursting thresholds of $P_{B U R S}$ and $P_{R B V}$, respectively. If either of these valves fails prematurely, then the propagation of the MCR is at risk of disruption. But because both valves are pneumatically connected by the air trapped within the air link, their pressures are additive, and hence the threshold for failure of either is the sum of the two. The condition for success is $Q_{F A I L}=0$, which during drainage of reservoir $n$ is satisfied if the pressure drop on the functional connection $P_{F C}=$ $Q_{F C} \times R_{F C}$ is (see also supplementary information for a detailed mathematical derivation):

$$
P_{F C(n)}<P_{B U R S(n)}+P_{R B V(n+1)} \quad \text { Equation } 1
$$


We calculated $P_{B U R S}$ (numerically) ${ }^{20}$ and $P_{R B V}$ (analytically, see Supplementary Information) for conduits with square cross section $(\mathrm{w}=\mathrm{h})$ for the typical dimension in our 3D printed CCs, and measured them experimentally for validation, Fig. 2b and Supplementary Fig. S4. Both $P_{B U R S}$ and $P_{R B V}$ are inversely proportional to the smallest dimension of the rectangular conduit. We accounted for the hydrophobic ceiling formed by the sealing tape in both cases (see Supplementary Fig. S2b), and which is a key feature to forming a functional SV. ${ }^{20}$ Note that because of the comparatively low pressures and small volume of the air links, the compressibility of air is negligible here.

Next, several MCRs featuring functional connections with large, and increasing $R_{F C}$ were tested with pumps with different capillary pressure and flow rates. The interplay between the resistance and the flow rate determines the operational window for the CDV while they are inversely proportional. We found excellent concordance between theory and experiments for the operation window of the MCR, and failure only occurred for the highest values of $R_{F C}$ (no. 5 and 6), and only for the most powerful capillary pumps, Fig. 2c,d and Supplementary Fig. S5. The

MCR designs used in the proof-of-concept applications shown below, are well within the failure 6 threshold, helping ensure reliable propagation of the chain reaction.
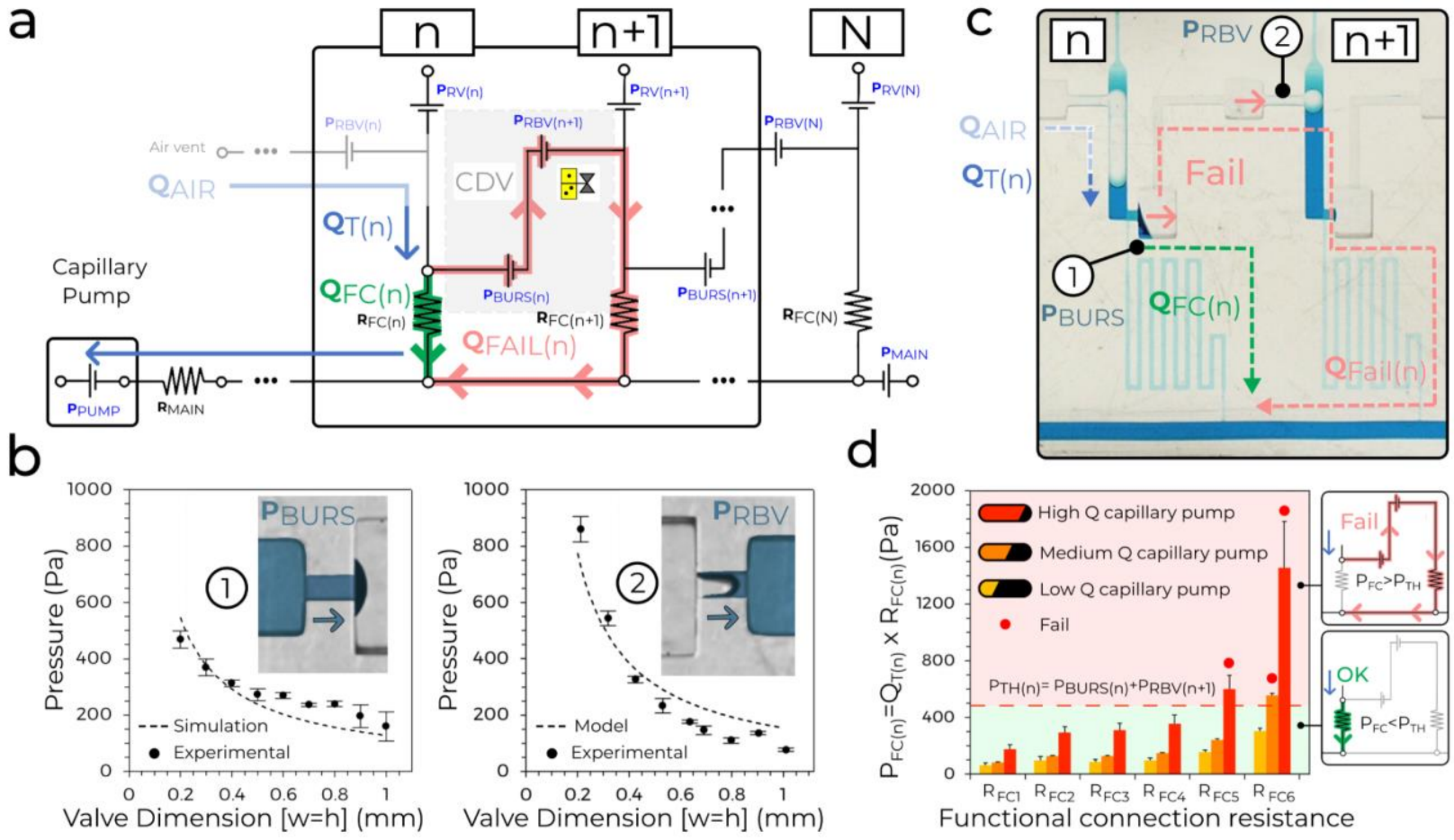

d

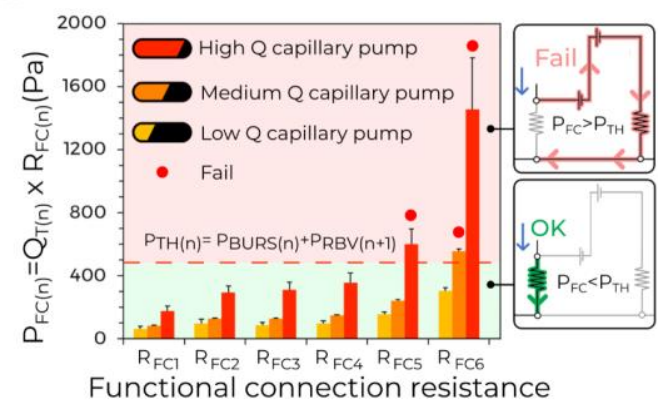


Fig. 2. Circuit analysis and experiments identify operational window for MCRs. (a) The simplified equivalent electrical circuit of the MCR units shown in Fig. 1. (b) Experimental SV burst pressure (1) and RBV retention pressure (2) for valves with conduits with different, square cross-sections fitted with a numerical and an analytical model, respectively. Error bars are standard deviations from 3 experiments. (c) Illustration of failure for a CDV with long serpentine functional connections with very high resistance leading to liquid breach inside the air link, and premature draining of reservoir $n+1$. (d) Tests of 6 MCRs with increasing $\mathrm{R}_{\mathrm{FC}}$ and three different paper pumps to determine the effect of varying the flow rate (n=3 for each paper pump and $R_{F C}$ ). As predicted, the CDVs fail when the pressure drop across the functional connection $P_{F C(n)}$ exceeds the CDV threshold pressure $P_{B U R S(n)}+P_{R B V(n+1)}$.

We designed a chip-to-chip interface with a leakage-free connection for liquid (main channel) and air (connecting the CDVs), respectively, and connected 4 chips with 75 MCR each, Fig. 3a and Video S2. This result illustrates the reliability of the MCR and of CDVs, and demonstrates integrated, large scale fluidic operations by 'passive' capillary microfluidics, beyond the capability of many 'active', computer programmable microfluidic systems.

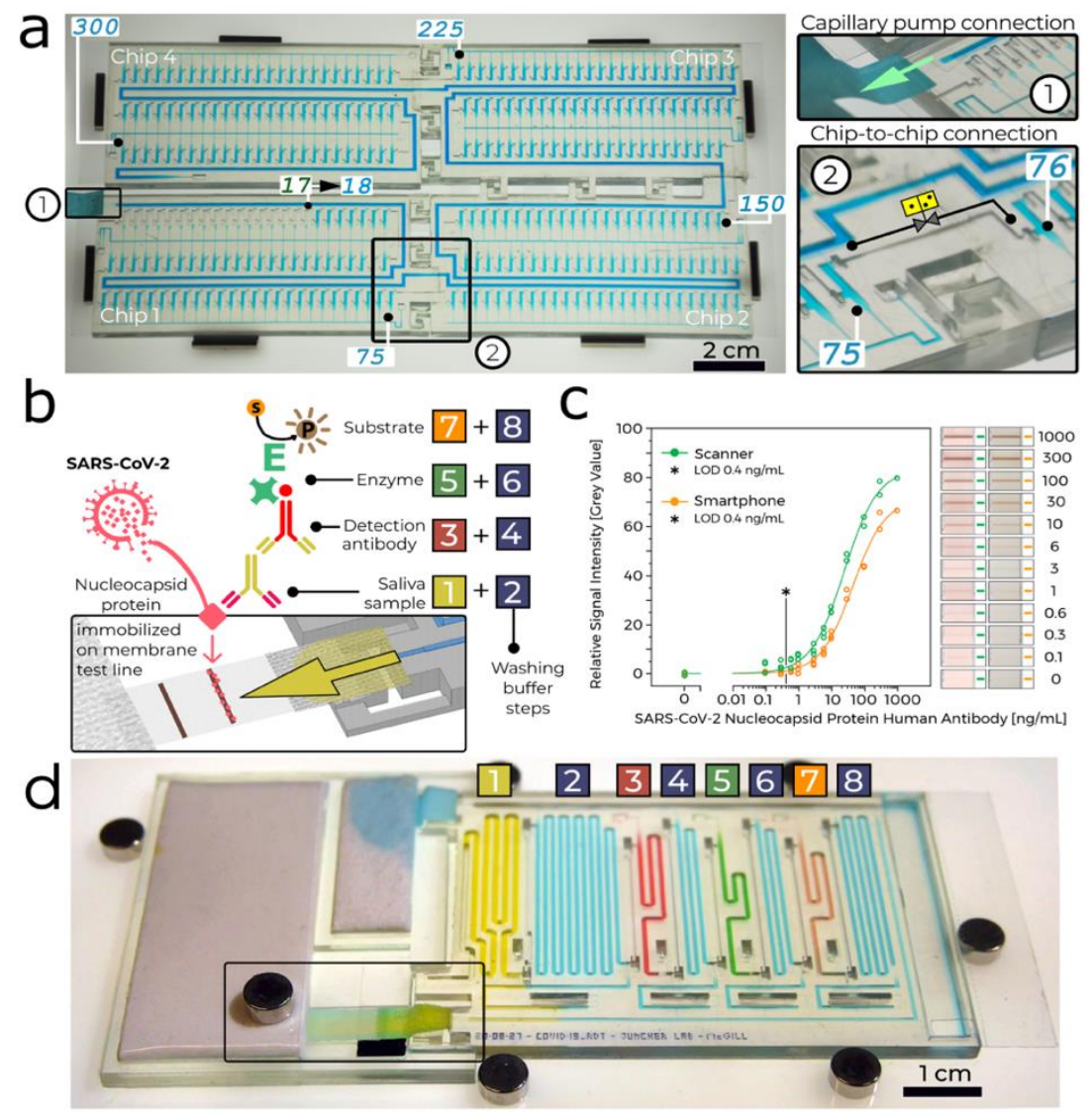


Fig. 3. Large-scale MCR and COVID-19 serology assay in saliva. (a) A MCR of 300 aliquots stored in $4.9 \mu 1$ reservoirs across four chained and interconnected chips. See Video S2. (b) SARS-CoV-2 antibody detection in saliva. Sequential, preprogrammed release of reagents via MCR is triggered by connecting the paper pump, see Video S3. The MCR supplies 4 reagents and 4 buffers in sequence. The functionality includes delivery and removal (by flushing) of solutions, metering $(40 \mu \mathrm{l}-200 \mu \mathrm{l})$ via reservoir size, flow speed and time control via the flow resistance of the functional connection and the capillary pressure of the paper pump. The enzymatic amplification produces a brown precipitate line visible to the naked eye. (c) Assay results and binding curve obtained by spiking antibody into saliva, and imaging by scanner and cell phone with representative images of the detection zone for each concentration, indicating the potential for quantitative point-of-care assays. (d) An assembled chip filled with colored solutions highlighting the channels for the different reagents and washing buffer.

\section{Automated SARS-CoV-2-specific saliva antibody detection assay}

We measured antibodies against the nucleocapsid protein (N protein) of SARS-CoV-2 in saliva, with application potential for early infection detection, ${ }^{21,22}$ initial patient assessment as prognosis indicator, ${ }^{23}$ and for serosurveys to differentiate vaccinated and naturally infected individuals. ${ }^{24}$ Conventional lateral flow assays with pre-dried reagents are simple to operate, but typically do not include enzymatic amplification that underlies the laboratory enzyme linked immunosorbent assay (ELISA), and have to be read-out within a few minutes of completion. Here, we used MCR to automate a sequence of 8 steps in common laboratory ELISA protocols, Fig. 3b. The chip is connected to a small paper pump to drain excess buffer, and a nitrocellulose strip for assay readout itself connected to a large-capacity paper pump that drives the MCR. Note that the MCR propagates in a direction opposite from the flow in the main channel, and reagents released sequentially from reservoirs all flow past previously emptied reservoirs, thus minimizing the diffusional mixing between reagents. We used 3,3'-Diaminobenzidine as a substrate which upon enzymatic conversion produced a brown, persisting precipitate that could serve both as an immediate readout and a record for archival. Assay parameters such as volume, time and reagent concentrations were optimized extensively following standard protocols (see Supplementary Fig. S6 for examples) and will be reported elsewhere. The result can be visualized by naked eye or quantified using a scanner or a smartphone integrated with a simple 
1 folded origami box to minimize light interference, with a sensitive, quantitative, and

2 reproducible output. (Fig. 3c and Supplementary Figs. S7 and S8).
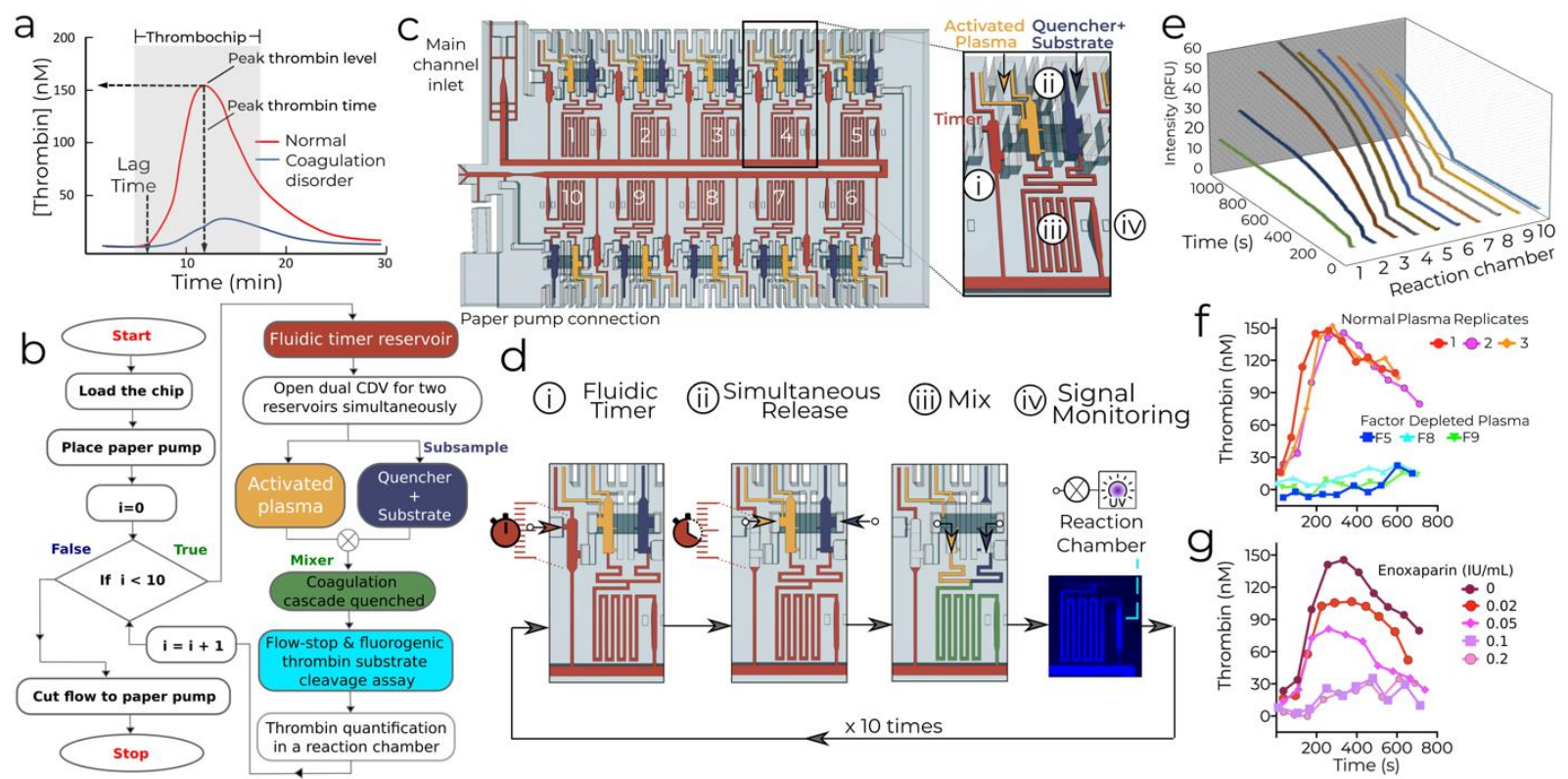

Fig. 4. Automated thrombin generation assay (TGA) by continuous analysis of plasma subsamples (thrombochip). (a) Model thrombin generation curve (thrombograms) for plasma with normal (red) and disordered (blue) coagulation. Grey box is the time window of the thrombochip. (b) TGA operations and algorithm encoded in the thrombochip. (c) Schematic of the thrombochip with inset and (d) showing (i) timer, (ii) simultaneous release of plasma and reagents (quencher and substrate), (iii) mixing, and (iv) flow-stop in the reaction chamber and monitoring of the fluorescence time-course signal. (e) Fluorescent thrombin substrate turnover in the ten 1 min-interval subsamples; the slope of each curve is proportional to thrombin concentration, and is one data point in the thrombogram. Abridged thrombograms of human plasma that is (f) normal (3 replicates of pooled plasma), factor-depleted (F5, F8, F9), and (g) mixed with anticoagulant drug (Enoxaparin) at different concentrations. The thrombin generation time-courses are concordant with expectations.

\section{Automated microfluidic thrombin generation assay}

Routine coagulation tests (prothrombin time, PT; and activated partial thromboplastin time, aPTT) are used as initial evaluation of hemostatic status. These tests terminate upon clot formation and thus only inform on the initiation of clotting; whereas the coagulation cascade continues and generates $95 \%$ of total thrombin (the final enzyme in the coagulation cascade). 
The hemostatic capacity, expressed as the endogenous thrombin potential, can therefore not be evaluated by these tests. Global coagulation assays, such as the thrombin generation assay (TGA) which provides the time course of active thrombin concentration in clotting plasma, are better measures of hemostatic function. Peak height, shape and area under curve of the thrombin generation curve (also known as the thrombogram, Fig. 4a), can be determined, and correlated to clinical phenotypes to investigate coagulation disorders, and measure the effect of anticoagulants. ${ }^{25}$ The first TGA was introduced in the $1950 \mathrm{~s}$, and involves the activation of coagulation of blood or plasma, followed by a two-stage assay that requires the collection and mixing of sub-samples with fibrinogen (or chromogenic substrates following their availability) at precisely timed intervals (e.g. $1 \mathrm{~min}$ ) over the course of $\sim 20 \mathrm{~min}$, followed by the quantification of thrombin in each of them. ${ }^{26,27}$ The labor intensity, strict timing requirements, and risk of error are great obstacles to wider adoption and clinical use of TGAs-by-subsampling. The calibrated automated thrombogram (CAT) introduced in 2002 simplifies operations thanks to newly synthesized thrombin substrates, a calibration TGA using the patient sample spiked with reference material, and mathematical extrapolation. ${ }^{28}$

Here, we demonstrate the capacity of MCR to automate the original TGA-bysubsampling in a 'thrombochip' - the first microfluidic implementation to the best of our knowledge. We devised an algorithm, Fig. 4b, for automating and timing the procedure with cascaded, iterative, and branching fluidic operations, Fig. 4c (and Supplementary Fig. S9) and structurally encoded it into a 3D printed chip (Video S5). Coagulation-activated plasma subsamples and reagent were loaded into the thrombochip, and upon triggering of the MCR, without further intervention, they were released at 1-min-intervals from the ten pairs of reservoirs, mixed in the serpentine mixer, and stored in a 2.1- $\mu 1$-reaction chamber with a width of $500 \mu \mathrm{m}$ for fluorescence signal generation and readout using a camera, Video S6. The concentration of thrombin in each of the subsamples is proportional to the rate of the fluorescence substrate turnover, and the time-course of thrombin is reported as a thrombogram.

Reliable execution of the TGA subsample analysis algorithm faced multiple practical challenges, and in particular draining of two reservoirs simultaneously is inherently unstable. Indeed, as soon as one reservoir starts being drained, the (absolute) pressure in the CC drops, and readily falls below the threshold of the RBV of the second reservoir, which will not burst, meaning the reservoir will remain filled. The MCR and 3D printing helped overcome this 
challenge and the reservoir pair containing plasma and reagents could be drained synchronously. An embedded air link connecting the outlet of reservoir $\mathrm{n}$ to the RBVs of both $n+1$ and $n+1$ ' which were identical and very weak RBVs (cross-section, $1 \times 1 \mathrm{~mm}^{2}$ ) lead to simultaneous bursting and reliable propagation of the chain reaction. Other critical features are a serpentine mixer; stop-flow and holding of the solution in the reaction chambers for the thrombin quantification; a pressure pinning structure at the main outlet to cut the hydraulic connection to the paper pump after completion of the fluidic operation; an RBV at the main outlet that pins liquid and helps prevent backflow to safeguards the reaction chambers from uncontrolled mixing and evaporation during the extended monitoring and imaging of the thrombin reaction.

As validation of the thrombochip, human pooled plasma, plasma depleted of Factor V, VIII and IX, and plasma spiked with the anticoagulant Enoxaparin (an anti-Factor Xa drug) were analyzed. The corresponding thrombograms were reproducible, consistent with normal and impaired coagulation cascades caused by factor depletion, and measured the dose-response of Enoxaparin, Fig. 4f, g. The general profile of the thrombograms generated in these proof-ofconcept experiments are comparable to those by CAT and other microtiter plate-based assays, ${ }^{29,30}$ but direct comparison of the data such as lag time and peak concentration requires standardized sample processing, reference materials and normalization, which can guide future development of the thrombochip.

\section{Conclusion and Discussion}

MCRs introduce deterministic, modular and programmable chain reactions at the mesoscale and constitute a new concept for autonomous, programmable liquid operations and algorithms via control of both hydraulic and pneumatic flow and connectivity. The automation of complex and repetitive liquid handling operations has so far only been possible with a computer, software programs, and cumbersome peripheral equipment, either robotics, or in the case of microfluidics, ${ }^{6}$ systems to supply reagents, power, or flow control. ${ }^{8-11}$ MCR introduces mesoscale chain reactions as a frugal, integrated, scalable, and programmable process that power integrated lab-on-a-chips.

The MCR chip micro-architecture is simultaneously the circuit and the code of the chain reaction, is manufacturable with a variety of techniques, and scalable along two distinctive paths: Firstly, following microelectronics example and Moore's law, by shrinking and increasing the 
number of features per unit area, and per unit volume (e.g. by using 3D printing). Secondly, by expanding the overall size of CC-MCRs by interconnecting and chaining chips, and, inspired by trees that draw liquids over $100 \mathrm{~m}$ in height, linking them to powerful capillary pumps. ${ }^{31} \mathrm{We}$ anticipate numbers of steps far beyond the 300 shown here, and much more complex algorithms than the ones of the thrombochip.

MCRs are generalizable, compatible with positive pressure operations, and could be interfaced with active microfluidics and robotic liquid handling systems. Spontaneous, capillaryflow MCRs may be further improved too with permanently hydrophilic resins or coatings, liquid storage pouches, and pre-dried reagents, ${ }^{32}$ notably for point-of-care applications, and any other uses. An end-user, by simply depositing a drop of solution at the inlet, could trigger a choreography of timed operations including aliquoting, delivery, mixing, flushing and reaction of multiple chemicals. As MCRs can be 3D printed and monolithically encoded in a chip, the entry barrier is very low (entry-level resin-based printers cost $<\$ 300$ ). MCRs may be homemanufactured easily, or mail-ordered, opening the way for rapid dissemination, and for new inventions, advances, and for downloadable and printable microfluidic apps.

\section{Data availability}

Data not presented in the article or supplementary material will be available upon request.

\section{References}

1 Denisov, E., Sarkisov, O. \& Likhtenshtein, G. I. Chemical Kinetics: Fundamentals and Recent Developments. (Elsevier, 2003).

2 NobelPrize.org. Award ceremony speech. Nobel Media AB 2021, <https://www.nobelprize.org/prizes/chemistry/1956/ceremony-speech/> (2021).

3 Mullis, K. et al. Specific enzymatic amplification of DNA in vitro: the polymerase chain reaction. Cold Spring Harb Symp Quant Biol 51 Pt 1, 263-273, doi:10.1101/sqb.1986.051.01.032 (1986).

4 Saiki, R. K. et al. Primer-directed enzymatic amplification of DNA with a thermostable DNA polymerase. Science 239, 487-491, doi:10.1126/science.2448875 (1988).

5 Dirks, R. M. \& Pierce, N. A. Triggered amplification by hybridization chain reaction. Proc Natl Acad Sci U S A 101, 15275-15278, doi:10.1073/pnas.0407024101 (2004).

6 Sackmann, E. K., Fulton, A. L. \& Beebe, D. J. The present and future role of microfluidics in biomedical research. Nature 507, 181-189, doi:10.1038/nature13118 (2014). 
7 Manz, A., Graber, N. \& Widmer, H. M. Miniaturized total chemical analysis systems: A novel concept for chemical sensing. Sens. Actuators B Chem. 1, 244-248, doi:10.1016/0925-4005(90)80209-i (1990).

8 Thorsen, T., Maerkl, S. J. \& Quake, S. R. Microfluidic large-scale integration. Science 298, 580-584, doi:10.1126/science.1076996 (2002).

9 Choi, K., Ng, A. H., Fobel, R. \& Wheeler, A. R. Digital microfluidics. Annu Rev Anal Chem 5, 413-440, doi:10.1146/annurev-anchem-062011-143028 (2012).

10 Strohmeier, O. et al. Centrifugal microfluidic platforms: advanced unit operations and applications. Chem Soc Rev 44, 6187-6229, doi:10.1039/c4cs00371c (2015).

11 Easley, C. J. et al. A fully integrated microfluidic genetic analysis system with sample-inanswer-out capability. Proc Natl Acad Sci U S A 103, 19272-19277, doi:10.1073/pnas.0604663103 (2006).

12 Olanrewaju, A., Beaugrand, M., Yafia, M. \& Juncker, D. Capillary microfluidics in microchannels: from microfluidic networks to capillaric circuits. Lab Chip 18, 23232347, doi:10.1039/c81c00458g (2018).

13 Olanrewaju, A. O., Robillard, A., Dagher, M. \& Juncker, D. Autonomous microfluidic capillaric circuits replicated from 3D-printed molds. Lab Chip 16, 3804-3814, doi:10.1039/c6lc00764c (2016).

14 Safavieh, R. \& Juncker, D. Capillarics: pre-programmed, self-powered microfluidic circuits built from capillary elements. Lab Chip 13, 4180-4189, doi:10.1039/c31c50691f (2013).

15 Ozer, T., McMahon, C. \& Henry, C. S. Advances in Paper-Based Analytical Devices. Annu Rev Anal Chem 13, 85-109, doi:10.1146/annurev-anchem-061318-114845 (2020).

16 Wang, X., Hagen, J. A. \& Papautsky, I. Paper pump for passive and programmable transport. Biomicrofluidics 7, 14107, doi:10.1063/1.4790819 (2013).

17 Kokalj, T., Park, Y., Vencelj, M., Jenko, M. \& Lee, L. P. Self-powered Imbibing Microfluidic Pump by Liquid Encapsulation: SIMPLE. Lab Chip 14, 4329-4333, doi:10.1039/c4lc00920g (2014).

18 Dal Dosso, F. et al. Creasensor: SIMPLE technology for creatinine detection in plasma. Anal Chim Acta 1000, 191-198, doi:10.1016/j.aca.2017.11.026 (2018).

19 Achille, C. et al. 3D Printing of Monolithic Capillarity-Driven Microfluidic Devices for Diagnostics. Adv Mater 33, e2008712, doi:10.1002/adma.202008712 (2021).

20 Glière, A. \& Delattre, C. Modeling and fabrication of capillary stop valves for planar microfluidic systems. Sens. Actuator A Phys. 130-131, 601-608, doi:10.1016/j.sna.2005.12.011 (2006).

21 Burbelo, P. D. et al. Sensitivity in Detection of Antibodies to Nucleocapsid and Spike Proteins of Severe Acute Respiratory Syndrome Coronavirus 2 in Patients With Coronavirus Disease 2019. J Infect Dis 222, 206-213, doi:10.1093/infdis/jiaa273 (2020).

22 Pisanic, N. et al. COVID-19 Serology at Population Scale: SARS-CoV-2-Specific Antibody Responses in Saliva. J Clin Microbiol 59, doi:10.1128/JCM.02204-20 (2020). 
23 Batra, M. et al. Role of IgG against N-protein of SARS-CoV2 in COVID19 clinical outcomes. Sci Rep 11, 3455, doi:10.1038/s41598-021-83108-0 (2021).

24 Beck, E. J. et al. Differentiation of SARS-CoV-2 naturally infected and vaccinated individuals in an inner-city emergency department. medRxiv, doi:10.1101/2021.10.13.21264968 (2021).

25 Binder, N. B. et al. Clinical use of thrombin generation assays. J Thromb Haemost 19, 2918-2929, doi:10.1111/jth.15538 (2021).

26 Macfarlane, R. G. \& Biggs, R. A thrombin generation test; the application in haemophilia and thrombocytopenia. J Clin Pathol 6, 3-8, doi:10.1136/jcp.6.1.3 (1953).

27 Pitney, W. R. \& Dacie, J. V. A simple method of studying the generation of thrombin in recalcified plasma; application in the investigation of haemophilia. J Clin Pathol 6, 9-14, doi:10.1136/jcp.6.1.9 (1953).

28 Hemker, H. C. et al. The calibrated automated thrombogram (CAT): a universal routine test for hyper- and hypocoagulability. Pathophysiol Haemost Thromb 32, 249-253, doi:10.1159/000073575 (2002).

29 Gerotziafas, G. T. et al. Comparison of the effect of fondaparinux and enoxaparin on thrombin generation during in-vitro clotting of whole blood and platelet-rich plasma. Blood Coagul Fibrinolysis 15, 149-156, doi:10.1097/00001721-200403000-00006 (2004).

30 Zavyalova, E. \& Kopylov, A. Exploring potential anticoagulant drug formulations using thrombin generation test. Biochem Biophys Rep 5, 111-119, doi:10.1016/j.bbrep.2015.11.011 (2016).

31 Wheeler, T. D. \& Stroock, A. D. The transpiration of water at negative pressures in a synthetic tree. Nature 455, 208-212, doi:10.1038/nature07226 (2008).

32 Gokce, O., Castonguay, S., Temiz, Y., Gervais, T. \& Delamarche, E. Self-coalescing flows in microfluidics for pulse-shaped delivery of reagents. Nature 574, 228-232, doi:10.1038/s41586-019-1635-z (2019).

Acknowledgments: We acknowledge Justin Lessard-Wajcer, Jay Pimprikar, Marziye Mirbagheri, Yiannis Paschalidis and Vahid Karamzadeh for their help, Yonatan Morocz for photography, and Thomas Gervais for insightful comments.

Funding: This work was supported by NSERC Strategic Project Grant STPGP 494495-16 and, NSERC Alliance Grant ALLRP 551058-20 and an McGill MI4 SCRF grant. M.Y. acknowledges FRQNT postdoctoral fellowship \#260284. A.S.K. acknowledges FRQNT postdoctoral fellowship (\#267919). D.J. acknowledges support from a Canada Research Chair in Bioengineering.

Author contributions: Methodology, Data curation, and Formal Analysis: D.J. A.O. and M.Y. designed and tested the two-layered MCR. M.Y. designed and tested the monolithic MCR. O.Y., A.P., J.R., Z.J., A.S. and A.P. designed MCR CC for experiments and analyzed the data for the COVID-19 antibody test. O.Y. performed the pressure characterization tests. M.Y. and A.N. performed the experiments and analyzed the data for the thrombochip. M.Y. designed and 
performed the experiments for the CDV failure. A.P. and A.S.K. performed the experiments for the 300 MCRs. M.Y. performed the CT scan and dimensions characterization. A.S. and A.P.

performed the contact angle measurements. G.K. performed the simulation for the burst pressure on COMSOL. D.J. guided design and analysis of all experiments. Visualization: M.Y, O.Y, A.N. and D.J. prepared the main figures. M.Y. and Z.J worked on the 3D visualizations. M.Y., O.Y., A.O., A.P., A.S.K, Z.J. and A.N. captured all the images and videos. G.K. and M.Y. worked on editing all the videos. Writing - original draft: M.Y., A.O., A.N. and D.J. Writing review \& editing: M.Y., O.Y., A.O., A.P., Z.J, J.R., G.K. A.N. and D.J. Project conception, administration, and supervision A.N. and D.J.

10 Competing interests: M.Y., A.O. and D.J. are inventors on a ROI submitted to McGill and 11 licensed. All other Authors declare no competing interests.

\section{Additional information:}

13 Supplementary Information is available for this paper.

14 Correspondence and requests for materials should be addressed to: David Juncker

6500-740 Dr Penfield Avenue
Montreal, QC. CA. H3A 0G1
david.juncker@ mcgill.ca
Phone: +1 (514) 398 7676, Fax: +1 (514) 3981790

Reprints and permissions information is available at www.nature.com/reprints 
Methods:

Chip design and fabrication

The chips were designed using AutoCAD (Autodesk) and exported as .STL files for 3D printing. CCs encoding MCRs were made with a digital micromirror display (DMD) 3D printer (Miicraft 100, Creative Cadworks, Toronto, Canada) using a transparent resin (Rapid Model Resin Clear, Monocure 3D, Regents Park, NSW, Australia) purchased from filaments.ca (Mississauga, Canada). The following printing parameters were used: The layer thickness was 20 $\mu \mathrm{m}$, the exposure time $1.5 \mathrm{~s}$ per layer, while the exposure time for the base layer was $10 \mathrm{~s}$ with 4 transition buffer layers. Following completion of the print, the chips were cleaned with isopropanol and post-cured for 1 min under UV (Professional CureZone, Creative Cadworks, Toronto, Canada)

Microchannels with cross-sections ranging from $250 \times 100 \mu \mathrm{m}^{2}$ to $1500 \times 1000 \mu \mathrm{m}^{2}$ were fabricated and hydrophilized by plasma activation for $10 \mathrm{~s}$ at $\sim 30 \%$ power (PE50 plasma chamber, Plasma Etch, Carson City, USA).

CCs were sealed with a delayed tack adhesive tape (9795R microfluidic tape, 3M) forming the cover.

\section{Paper capillary pump}

Filter papers (Whatman filter paper grade 4, 1 and 50 Hardened, Cytiva, Marlborough, USA) were used as paper capillary pumps for all experiments except the SARS-CoV-2 antibody assay. The pore size from 4,1 , and 50 hardened is in decreasing order, and flow resistance and capillary pressure increase with decreasing pore size.

For the SARS-CoV-2 antibody assay, absorbent pads (Electrophoresis and Blotting Paper, Grade 238, Ahlstrom-Munksjo Chromatography, Helsinki, Finland) were used as pump.

\section{Chip-to-chip connections for the 300 capillary flow events}

To obtain a leakage-free connection, a thin layer of uncured photoresin, prepared by mixing poly (ethylene glycol) diacrylate (PEG-DA MW 258, Sigma Aldrich) and Irgacure-819 (1\% w/w), was applied to all of the chip-to-chip interfaces. Next, the chips were assembled and exposed to UV light for in a UV chamber (320-390 nm, UVitron Intelliray 600 at 50\% intensity) for $30 \mathrm{~s}$ to cure the resin and seal the connections.

\section{Videos and image processing}

Videos and images were recorded using a Panasonic Lumix DMC-GH3K. Structural images of the chip and the embedded conduits were obtained using a micro CT (Skyscan 1172, Bruker, Billerica, USA) and used to confirm the dimensions. Contact angles were measured based on side view images $(n=3)$ and analyzed using the Dropsnake extension in Image J.

\section{Modeling and Calculations}

The theoretical burst pressures of capillary stop valves were calculated by solving the flow field using the finite element method (FEM) with COMSOL Multiphysics 5.5 (Burlington, MA). Experimentally measured contact angles $\left(100^{\circ}\right.$ and $40^{\circ}$ for the cover and the channel, respectively) were used to solve two-phase capillary flow using the level-set method. The capillary flows leading up to the stop valve was solved for a time period of $0-0.02$ seconds with a time step of $1 \times 10^{-5} \mathrm{~s}$. The inlet pressure was varied with $10 \mathrm{~Pa}$ increment for each simulation, until burst was observed. 


\section{Experiments on pressure thresholds for capillary stop valve and retention burst valve}

We 3D-printed modules to evaluate SV/RBV with different cross section areas. Each module contained three SV/RBV for replicate results. SV/RBV consisted of a two-level stop valve based on a geometrical channel expansion, as described elsewhere. ${ }^{12}$ The chips integrated a conical inlet/outlet for tubing connection to a Microfluidic Flow Controller System (MFCS-4C) and Fluiwell package (Fluigent, Paris France) with fluidic reservoirs containing 5\% red food dye in MilliQ water solution (see Supplementary Fig. S4 for setup images and S2 for contact angles). MAESFLO 3.3.1 software (Fluigent, Paris France) controlled the application of positive or negative pressure to calculate the burst pressures of the stop valve (liquid burst into air link) and retention burst valve (receding meniscus), with increments of 10 mbar ( 1000 Pa).

\section{$\underline{\text { SARS-CoV-2 antibody assay }}$}

\section{Reagents}

SARS-CoV-2 nucleocapsid protein was purchased from Sino Biological, Inc., Beijing, China (40588-V08B). Human Chimeric antibody against SARS-CoV-2 nucleocapsid protein was purchased from Genscript Biotech, Piscataway, USA (A02039). SIGMAFAST TM 3,3'Diaminobenzidine (DAB) tablets were purchased from Sigma-Aldrich, Oakville, Canada. Biotinylated Goat-anti-Human antibody was purchased from Cedarlane, Burlington, Canada (GTXHU-003-DBIO). Pierce ${ }^{\text {TM }}$ Streptavidin Poly-HRP (21140) was purchased from ThermoFisher, Ottawa, Canada.

\section{Nitrocellulose strips}

Nitrocellulose membranes (Whatman FF80HP Plus nitrocellulose-backed membranes, Cytiva, USA) were cut into $5.2 \mathrm{~mm}$ wide strips using the Silhouette Portrait paper cutter (Silhouette, Lindon, USA). Membranes were striped with a $5 \mathrm{~mm}$ wide test line of $0.25 \mathrm{mg} / \mathrm{mL}$ SARS-CoV-2 nucleocapsid protein delivered using a programmable inkjet spotter (sciFLEXARRAYER SX, Scienion, Berlin, Germany). The test line consists of 4 lanes of 50 droplets of about $350 \mathrm{pL}$ printed $100 \mu \mathrm{m}$ apart from each other. 8 passes of 25 droplets were used for each lane on even and odd positions to allow solution absorption in between passes. The membranes were then dried for $1 \mathrm{~h}$ at $37^{\circ} \mathrm{C}$ before blocking by dipping into $1 \%$ BSA in 1X PBS solution until completely wet, then retrieved and left to dry for $1 \mathrm{~h}$ at $37^{\circ} \mathrm{C}$ and then stored with desiccant at $4{ }^{\circ} \mathrm{C}$ until use the next day.

\section{Connection of capillary pump and nitrocellulose chip to MCR chips}

Nitrocellulose strips were mounted following standard lateral flow assay assembly protocols. The nitrocellulose was connected to glass fiber conjugate pad (G041 SureWick, Millipore Sigma, Oakville, Canada) on one end, and to an absorbent pad (Electrophoresis and Blotting Paper, Grade 238, Ahlstrom-Munksjo Chromatography, Helsinki, Finland) serving as the capillary pump at the other end. All three were attached to an adhesive tape serving as the backing layer. For the saliva antibody assay, the nitrocellulose strip was sandwiched between three absorbent pads $\left(15 \times 25 \mathrm{~mm}^{2}\right)$ and clamped with a paper clip. For the food-dye demonstrations a single absorbent pad $\left(25 \times 45 \mathrm{~mm}^{2}\right)$ was magnetically clamped to the nitrocellulose membrane.

$\underline{\text { Saliva assay protocol }}$ 
Human saliva was extracted with oral swabs (SalivaBio, Salimetrics, State College, USA), followed by centrifugation and 1:10 dilution with $0.22 \mu \mathrm{m}$ filtered phosphate buffer saline containing $1 \%$ BSA, $0.1 \%$ Tween 20 . Human chimeric antibody against SARS-CoV-2 nucleocapsid protein at 0 to $1000 \mathrm{ng} / \mathrm{ml}$ was spiked into diluted saliva and loaded to the sample reservoirs. 3 replicate measurements for concentrations of $0-10 \mathrm{ng} / \mathrm{ml}, 2$ replicate measurements for concentrations of $30-300 \mathrm{ng} / \mathrm{ml}$, and one measurement for $1000 \mathrm{ng} / \mathrm{ml}$. Control line (CL) in the nitrocellulose strip confirms reagents delivery and colorimetric reaction completion.

Image analysis on the nitrocellulose strips

After drainage of all reservoirs, the nitrocellulose membrane strip was removed, placed on a support, and let dry for one hour.

The dry strips were imaged using (i) a flatbed scanner (mfc-9970cdw, Brother) at a resolution of 600 dpi and (ii) using a Huawei P10 smartphone with a 12-megapixel image sensor and a rear camera with a $27-\mathrm{mm}$ focal length (Huawei, Shenzhen, China) within a customized box. The box was cut and folded with black cardboard paper to block ambient light when imaging with the smartphone. The box had two slots fitting the size of camera and nitrocellulose strip, respectively, to ensure accurate alignment of strip for readout. Images were taken with oncamera dual tone LED flash on at full power. Analysis of smartphone-taken and scanned images was done as follows.

Mean grey values of nitrocellulose test lines (TL) were extracted with ImageJ $1.48 \mathrm{v}$ (ImageJ, public domain software, Wayne Rasband, National Institutes of Health, USA) within a $100 \times 10$ pixels rectangular area. Local background grey values were taken at 0.1 inch $(2.5 \mathrm{~mm})$ above each test line (following direction of the flow) for the same rectangular area, and subtracted from test line values. The normalized standard curve was then generated by subtracting negative control signal value $(0 \mathrm{ng} / \mathrm{mL})$ from all data points.

The LOD was calculated using the 3-sigma criterion using a nonlinear four-parameter logistic curve fit of the log-transformed data with OriginPro 8.5 SPR (OriginLab Corporation, Nothampton, MA).

Automated microfluidic thrombin generation assay (Thrombochip)

Citrated human plasma, fluorogenic thrombin substrate Z-GGR-AMC and Enoxaparin were purchased from Sigma-Aldrich, Oakville, Canada; Batroxobin was from Prospec, East Brunswick, USA; Technothrombin TGA RC High reagent was from Diapharma, West Chester, USA; Human thrombin, non-patient plasma that were immuno-depleted of Factor V and Factor IX, and FVIII inactivated were from Haematologic Technologies, Essex Junction, USA; (4-(2hydroxyethyl)-1-piperazineethanesulfonic acid) (HEPES), Ethylenediaminetetraacetic acid (EDTA), $\mathrm{CaCl} 2$ were from Sigma-Aldrich, Oakville, Canada.

The purchased pooled human plasma was prepared by the manufacturer from whole blood collected by standard industry method using $4 \%$ trisodium citrate as an anticoagulant, pooled and then centrifuged. The resulting plasma was $0.45 \mu \mathrm{m}$ filtered and lyophilized. Factor V- and Factor IX-depleted plasma were immune-depleted; Factor VIII-depleted plasma was prepared by chemical depletion. The plasma preparations were assayed to ensure the activity of the remaining factors by the manufacturer.

Human plasma (pooled normal or factor-depleted) were defibrinated by the addition of batroxobin (final concentration $0.6 \mathrm{BU} / \mathrm{mL}$ ). The mixtures were incubated at room temperature 
for $20 \mathrm{~min}$, followed by an additional incubation at $4{ }^{\circ} \mathrm{C}$ for $1 \mathrm{~h}$. The mixtures were then centrifuged at 10,000-g for 10 min to remove the fibrin clot and other debris. Defibrinated plasma were collected from the supernatant.

A solution containing $21 \%$ defibrinated plasma (plasma defibrination is needed to prevent clogging of the microfluidic channels by the fibrin clot), $48 \%$ Technothrombin TGA RC High reagent (high phospholipid and relipidated tissue factor content) and $20 \mathrm{mM} \mathrm{CaCl}_{2}$ in $25 \mathrm{mM}$ HEPES at $\mathrm{pH} 7.4$ was loaded into the sample reservoirs of the thrombochip. A substrate solution containing $420 \mu \mathrm{M}$ Z-GGR-AMC, $30 \mathrm{mM}$ EDTA in $25 \mathrm{mM}$ HEPES at pH 7.4 was loaded into the reagent reservoirs. The concentration of plasma, activation agent and substrate were optimized to yield a peak thrombin level and time of $150 \mathrm{nM}$ and $200 \mathrm{~s}$. All solutions were equilibrated to room temperature for 20 min before loading. Coagulation-inhibited plasma contained Enoxaparin at final concentrations of 0 to 1.0 anti-Xa units $/ \mathrm{mL}$ or IU/mL. The samples and reagents were loaded on the chip after initiating the coagulation cascade. The paper pump was connected to the chip to start the flow after 5 min from initiating the coagulation cascade. Fluorescence signals generated in the reaction chambers were monitored by illuminating the thrombochip with UV light at $365 \mathrm{~nm}$ with $20 \mathrm{~W}$ (realUVTM LED Flood Light, Waveform Lighting, USA) and the visible $440 \mathrm{~nm}$ fluorescence emission signals measured by imaging at $5 \mathrm{~s}$ intervals using a Panasonic Lumix DMC-GH3K digital camera (f/3.5, Exposure time: 2 s, ISO200). The rate of fluorescence signal generation in each reaction chamber (i.e. the slope of the recorded fluorescence generation curve) is a measure of the rate of substrate turnover by thrombin and was used to deduce the amount of thrombin generated using a standard curve. Image $\mathbf{J}$ was used to analyze the images for fluorescence intensity.

\section{Standard curve for thrombin quantification}

10 human thrombin solutions at concentrations ranging from 0 to $300 \mathrm{nM}$ in $25 \mathrm{mM}$ HEPES at $\mathrm{pH} 7.4$ were loaded into the 10 sample reservoirs in the thrombochip. A substrate solution containing $420 \mu \mathrm{M}$ Z-GGR-AMC, $30 \mathrm{mM}$ EDTA in 25mM HEPES at pH 7.4 was loaded into the reagent reservoirs. The standard curve was constructed by plotting the slope of the recorded fluorescence generation curve in each reaction chamber against the known thrombin concentration of the solution that was loaded to the corresponding sample reservoir. 